\title{
MEANS AND VARIANCES OF STOCHASTIC VECTOR PRODUCTS WITH APPLICATIONS TO RANDOM LINEAR MODELS*
}

\author{
GERALD G. BROWN† AND HERBERT C. RUTEMILLER $\$$
}

\begin{abstract}
Applications in operations research often employ models which contain linear functions. These linear functions may have some components (coefficients and variables) which are random. (For instance, linear functions in mathematical programming often represent models of processes which exhibit randomness in resource availability, consumption rates, and activity levels.) Even when the linearity assumptions of these models is unquestioned, the effects of the randomness in the functions is of concern. Methods to accomodate, or at least estimate for a linear function the implications of randomness in its components typically make several simplifying assumptions. Unfortunately, when components are known to be random in a general, multivariate dependent fashion, concise specification of the randomness exhibited by the linear function is, at best, extremely complicated, usually requiring severe, unrealistic restrictions on the density functions of the random components. Frequent stipulations include assertion of normality, or of independence-yet, observed data, accepted collateral theory and common sense may dictate that a symmetric distribution with infinite domain limits is inappropriate, or that a dependent structure is definitely present. (For example, random resource levels may be highly correlated due to economic conditions, and nonnegative for physical reasons.) Often, an investigation is performed by discretizing the random components at point quantile levels, or by replacing the random components by their means-methods which give a deterministic "equivalent" model with constant terms, but possibly very misleading results. Outright simulation can be used, but requires considerable time investment for setup and debugging (especially for generation of dependent sequences of pseudorandom variates) and gives results with high parametric specificity and computation cost. This paper shows how to use elementary methods to estimate the mean and variance of a linear function with arbitrary multivariate randomness in its components. Expressions are given for the mean and variance and are used to make Tchebycheff-type probability statements which can accomodate and exploit stochastic dependence. Simple estimation examples are given which lead to illustrative applications with (dependent-) stochastic programming models.
\end{abstract}

\section{Introduction}

Mathematical models in operations research often require the calculation of quantities which are sums of products of random variables. (For example, see the realistic view of stochastic linear programming taken by Tintner [15].) Sums of products of random variables are the generic structural elements fundamental to linear models exhibiting complex randomness.

In the interest of tractable mathematics, it is commonly assumed either that the random variables may be treated as constants, or replaced by their expectations. Even where these simplifying assumptions are not made, severe parametric assumptions, e.g., normality, pairwise independence, etc., are usually required to achieve analytic probability statements concerning the function of interest. Other approaches use weighted sums of uniform, exponential or other variates in approximation schemes, or use characteristic functions of component densities to carry out the indicated transformation and attempt inversion of the result. Finally, Monte Carlo solutions to this problem may be achieved, but are of limited value due to the parametric specificity

*Accepted by Marcel F. Neuts; received September 2, 1975. This paper has been with the authors 5 months, for 3 revisions.

† Naval Postgraduate School, Monterey.

$\ddagger$ California State University, Fullerton. 
and high cost of results. For excellent examples of these methods see Jagannathan [7], King, Sampson and Simms [8], Miller and Wagner [10], Searle [12], and Sengupta [13], [14].

This paper addresses the problem of determining the mean and variance of sums of products of random variables in the most general linear case, i.e., arbitrary correlated multivariate densities for all components in the model. The results obtained are useful for making nonparametric Tchebycheff-type probability statements. Particular examples are given which use these probability statements as chance constraints in stochastic linear programming.

Simplifications of this model for moments of weighted sums of random variables have been presented by several authors. For instance, the highly successful heuristic given by Graves and Whinston [5] for the quadratic assignment problem is based on such a derivation.

\section{Notation and Development}

Let $b$ and $c$ be two random $n$-element column vectors. We denote the expectation of these vectors as

$$
E\left(\begin{array}{l}
b \\
c
\end{array}\right)=\mu=\left(\begin{array}{l}
\mu_{b} \\
\mu_{c}
\end{array}\right)
$$

The symbols $\tilde{b}$ and $\tilde{c}$ will indicate the deviation of each random vector from its expectation. The variance-covariance matrix will be signified by

$$
E\left(\begin{array}{l}
\tilde{b} \\
\tilde{c}
\end{array}\right)\left(\begin{array}{l}
\tilde{b} \\
\tilde{c}
\end{array}\right)^{\prime}=\Sigma=\left(\begin{array}{ll}
\Sigma_{b b} & \Sigma_{b c} \\
\Sigma_{c b} & \Sigma_{c c}
\end{array}\right),
$$

while the symbol $\Sigma^{*}$ will signify this matrix with $b$ and $c$ interchanged.

Let $q$ be a random scalar variable, defined as the inner product of these random vectors:

$$
q=b^{\prime} c
$$

Expressions for the mean, $E(q)$, and variance, $V(q)$, in terms of the moments of $b$ and $c$ are derived in the Appendix. They are:

$$
E(q)=\mu_{b}^{\prime} \mu_{c}+\operatorname{Tr} \Sigma_{b c}
$$

where $\operatorname{Tr} \Sigma_{b c}$ indicates the trace, or sum of the diagonal elements of $\Sigma_{b c}$;

$$
V(q)=\mu^{\prime} \Sigma^{*} \mu+2 \mu_{b}^{\prime} E\left(\tilde{c} \tilde{c}^{\prime} \tilde{b}\right)+2 \mu_{c}^{\prime} E\left(\tilde{b} \tilde{b}^{\prime} \tilde{c}\right)+E\left(\tilde{b}^{\prime} \tilde{c} \tilde{c}^{\prime} \tilde{b}\right)-\left(\operatorname{Tr} \Sigma_{b c}\right)^{2} .
$$

(2) and (3) represent the most general outcome, where any element of $b$ may be correlated with any other elements of $b$ or $c$. Two special cases occur frequently. First, suppose that the elements of $b$ are correlated with one another, the elements of $c$ are correlated with one another, but that $b$ and $c$ are stochastically independent. Then

$$
\begin{gathered}
\sum=\left(\begin{array}{cc}
\Sigma_{b b} & 0 \\
0 & \Sigma_{c c}
\end{array}\right), \text { and } \\
E(q)=\mu_{b}^{\prime} \mu_{c}, \\
V(q)=\mu_{b}^{\prime} \Sigma_{c c} \mu_{b}+\mu_{c}^{\prime} \Sigma_{b b} \mu_{c}+\operatorname{Tr}\left(\Sigma_{b b} \Sigma_{c c}\right) .
\end{gathered}
$$

Secondly, we note that, if all elements of both matrices are stochastically independent, $\Sigma$ is a diagonal matrix, and computation of (5) is greatly simplified. 
An assumption frequently made is that elements of vectors $b$ and $c$ have a multivariate normal distribution. If we adopt this parametric condition, then, even in the general case where $b$ and $c$ are correlated, (3) is greatly simplified. For multivariate normal distributions, $E\left(\tilde{b} \tilde{b}^{\prime} \tilde{c}\right)=E\left(\tilde{c} \tilde{c}^{\prime} \tilde{b}\right)=0$, and all higher moments are expressible in terms of the first two moments [1, p.38]. (3) becomes

$$
V(q)=\mu^{\prime} \Sigma^{*} \mu+\operatorname{Tr}\left(\Sigma_{b b} \Sigma_{c c}\right)+\operatorname{Tr}\left(\Sigma_{b c}^{2}\right)
$$

\section{An Estimation Example}

A job shop is bidding on the manufacture of units which will consume two raw materials and skilled labor. Let $b$ be the vector of material and labor requirements per unit of product, and $c$ the vector of respective costs per unit of $b$. Then the total manufacturing cost per unit, $q$, may be computed with (1). Due to unknown factors in the manufacturing process (e.g., proportion of scrap units produced) the elements of $b$ are random variables. Further, although the skilled labor cost is fixed by contract, other elements of $c$ are random variables dependent upon commodity prices and other factors.

Historical cost accounting data are available for similar products in the form of job orders and associated material requisitions and labor charges, and are used as prior information to determine the means and variance-covariance matrices of $b$ and $c$ as follows:

$$
\begin{aligned}
\mu_{b} & =\left[\begin{array}{l}
4.2 \text { units of I } \\
6.8 \text { units of II } \\
4.8 \text { hours of labor }
\end{array}\right), \\
\mu_{c} & =\left(\begin{array}{l}
\$ 2.15 \text { per unit of I } \\
\$ 1.50 \text { per unit of II } \\
\$ 6.20 \text { per hour }
\end{array}\right], \\
\Sigma_{b b} & =\left(\begin{array}{lll}
0.7 & 0.8 & 0.2 \\
0.8 & 1.2 & 0.3 \\
0.2 & 0.3 & 0.5
\end{array}\right) \\
\Sigma_{c c} & =\left(\begin{array}{lll}
0.6 & 0.1 & 0.0 \\
0.1 & 0.2 & 0.0 \\
0.0 & 0.0 & 0.0
\end{array}\right) \\
\Sigma_{b c} & =\mathbf{0}
\end{aligned}
$$

Prices of the two raw materials are positively correlated, as are the amounts consumed and the labor expended. In this example, there is no correlation between commodity prices and quantities consumed during manufacture, so (4) and (5) are the appropriate equations.

$$
\begin{aligned}
E(q) & =\mu_{b}^{\prime} \mu_{c}=\$ 48.99 \\
V(q) & =\mu_{c}^{\prime} \Sigma_{b b} \mu_{c}+\mu_{b}^{\prime} \Sigma_{c c} \mu_{b}+\operatorname{Tr}\left(\Sigma_{b b} \Sigma_{c c}\right) \\
& =41.23+25.54+0.82 \\
& =67.59 .
\end{aligned}
$$

Hence, the expected cost for one completed unit is $\$ 48.99$ with a standard deviation of $\$ 8.22$.

The (unknown) probability distribution of $q$ depends upon the mathematical form 
of the multivariate density functions of $b$ and $c$, and the transformation, (1). However, the moments calculated above are exact, and we may use them to make nonparametric statements about the distribution of $q$. Two illustrations follow.

\section{Tchebycheff's Inequality}

Regardless of the underlying distributions, at least $1-1 / k^{2}$ of the observations of $q$ will be within $k$ standard deviations of $E(q)$; e.g., there is a 75 percent probability that the price will lie in the interval \$32.55-65.43.

\section{Camp-Meidell Inequality}

Some mild assumptions about the distribution of $q$ lead to much tighter bounds [2]. If we assume that the distribution is unimodal, with the mode occurring at $E(q)$, then at least $1-1 / 2.25 k^{2}$ of the observations will lie within $k$ standard deviations of the mean; e.g., there is an 89 percent probability that the price will lie in the interval $\$ 32.55-65.43$.

For more general treatments of Tchebycheff-type bounds, see [4] and [9].

\section{Linear Programming Applications}

The chance constrained programming model of Charnes and Cooper [3] presents interesting applications of these techniques. A deterministic linear program may be stated

$$
\operatorname{Max} c^{\prime} x, \quad \text { s.t. } A x \leqslant b,
$$

with $A$ an $m \times n$ constant matrix of technological coefficients, $c$ a vector of constant prices and $b$ a vector of constant resource levels. If we change the sense of $b$ to that of a vector of resource levels realized from a multivariate density function, $f(b)$, then a reformulation of (7) yields

$$
\operatorname{Max} c^{\prime} x, \quad \text { s.t. } \operatorname{Pr}[A x \leqslant b] \geqslant \alpha,
$$

where $\alpha$ is a set of probabilities of individually satisfying each of the $m$ constraints. This problem may be solved by using the density function asserted for $b$ to find a set of bounds, $\beta(\alpha, f(b))$, such that $\operatorname{Pr}[b \geqslant \beta] \geqslant \alpha$, and, if $f(b)$ is free of terms involving $x$, reformulating $(8)$ to the "deterministic equivalent" form

$$
\operatorname{Max} c^{\prime} x \text {, s.t. } A x \leqslant \beta .
$$

Alternatively, let $\dot{\alpha}$ be the scalar joint probability that solutions of (9) will ultimately prove simultaneously feasible for all constraints. If the variates $b$ are independent, $\dot{\alpha}=\prod_{j=1}^{m} \alpha_{j}$, but if the terms in $b$ are correlated, extraction of $\dot{\alpha}$ from $f(b)$ may, or may not, be possible or practical depending on the mathematical form of $f(b)$, the dimensionality of $b$, and so forth.

If $f(b)$ is unknown or mathematically uncooperative we may use the moments of the vector $b$ to specify $\beta$ nonparametrically with Tchebycheff's inequalities. For instance

$$
\operatorname{Pr}\left[b_{j}<\mu_{j}-k_{j}\left(\sigma_{j j}\right)^{1 / 2}=\beta_{j}\right]=1-\alpha_{j} \leqslant\left(k_{j}^{2}\right)^{-1}, \quad j=1,2, \ldots, m .
$$

Also, a frequent and reasonable simplification is $k_{1}=\cdots=k_{m}=k$.

Further, we can specify a bound for $\dot{\alpha}$ by using a multivariate Tchebycheff inequality.

$$
\operatorname{Pr}\left[b_{j}<\mu_{j}-k_{j}\left(\sigma_{j j}\right)^{1 / 2}=\beta_{j} ; j=1, \ldots, m\right]=1-\dot{\alpha} \leqslant \sum_{j=1}^{m}\left(k_{j}^{2}\right)^{-1},
$$




$$
\begin{aligned}
\text { or, for } k_{1}=\cdots= & k_{m}=k \\
& \operatorname{Pr}\left[b<\mu-k(\operatorname{diag} \Sigma)^{1 / 2}=\beta\right]=1-\dot{\alpha} \leqslant m / k^{2} .
\end{aligned}
$$

Here, $(\operatorname{diag} \Sigma)^{1 / 2}$ denotes a column vector with components $\left(\sigma_{j j}\right)^{1 / 2}$.

Another Techebycheff inequality presented by Olkin and Pratt [11] can be used to specify a bound for $\dot{\alpha}$. If we use $\Pi$ to represent the correlation matrix of $b$ so that $\Pi_{j k}=\sigma_{j k} /\left(\sigma_{j j} \sigma_{k k}\right)^{1 / 2}$, and simplify the presentation by choosing the constants $k_{1}$ $=\cdots=k_{m}=k$, then

$$
\begin{aligned}
\operatorname{Pr}\left[b<\mu-k(\operatorname{diag} \Sigma)^{1 / 2}\right. & =\beta]=1-\dot{\alpha} \\
& \leqslant\left(k^{2} m^{2}\right)^{-1}\left[(\rho)^{1 / 2}+\sqrt{\left(m^{2}-\rho\right)(m-1)^{1 / 2}}\right]^{2}, \text { with }
\end{aligned}
$$

$\rho=e^{\prime} \Pi e$, where $e$ is a vector of l's.

\section{A Linear Programming Example}

To illustrate, using $b$ from the example in $\S 3$,

$$
\rho=\left(\begin{array}{lll}
1 & 1 & 1
\end{array}\right)\left(\begin{array}{lll}
1.0 & 0.87 & 0.34 \\
0.87 & 1.0 & 0.39 \\
0.34 & 0.39 & 1.0
\end{array}\right)\left(\begin{array}{l}
1 \\
1 \\
1
\end{array}\right)=6.20
$$

$1-\dot{\alpha} \leqslant 2.62 / k^{2}$ by use of (12). We note that this is a better bound than the $3 / k^{2}$ produced by (11).

For $k=3$ we find

$$
\begin{aligned}
\dot{\alpha} & \geqslant 0.71 \\
\beta=\mu-k(\operatorname{diag} \Sigma)^{1 / 2}= & \left(\begin{array}{l}
4.2-3(0.84) \\
6.8-3(1.10) \\
4.8-3(0.71)
\end{array}\right)=\left(\begin{array}{l}
1.68 \\
3.50 \\
2.67
\end{array}\right) .
\end{aligned}
$$

If (7) is further generalized to allow both $b$ and $c$ to be random vectors, possibly correlated, we can easily incorporate the expectation of the objection function in a deterministic equivalent. Alternately, following the development of [3], $x=D b$, with $D$ an $n \times m$ matrix, we reformulate (7) to

$$
\operatorname{Max} E\left[c^{\prime} x\right], \quad \text { s.t. } \operatorname{Pr}[A x \leqslant b] \geqslant \alpha, \quad x=D b,
$$

which by substitution becomes

$$
\operatorname{Max} E\left[c^{\prime} D b\right], \quad \text { s.t. } \operatorname{Pr}[A D b \leqslant b] \geqslant \alpha .
$$

Using the notation and results of $\S 2$, we derive for the objective function: $E\left[c^{\prime} D b\right]$ $=\mu_{c}^{\prime} D \mu_{b}+\operatorname{Tr}\left\{D \Sigma_{b c}\right\}$. The constraint set may be restated

$$
\begin{aligned}
\operatorname{Pr}[A D b \leqslant b] & =\operatorname{Pr}[A D b-b \leqslant 0] \\
& =\operatorname{Pr}[(A D-I) b \leqslant 0] \geqslant \alpha .
\end{aligned}
$$

Thus, using the moments of $b$ it follows [1, p. 24] that the vectors of expectations and variances are

$$
\begin{aligned}
E[(A D-I) b] & =(A D-I) \mu_{b}=\delta(D) \\
V[(A D-I) b] & =\operatorname{diag}\left\{(A D-I) \Sigma_{b b}(A D-I)^{\prime}\right\} \\
& =\gamma(D)
\end{aligned}
$$


The procedure, then, is as follows:

(1) Specify an acceptable $\alpha_{j}$, the probability of feasibility for each constraint.

(2) Use (10), (11), or (12) to generate the set of $k$ 's and $\dot{\alpha}$. A set of slack variables, $\nu$, is introduced. Adopting the symbol "*" to represent congruent multiplication of two commensurate matrices [6, p. 549 ff.], the deterministic equivalent programming model for (13) becomes

$$
\begin{gathered}
\operatorname{Max} \mu_{c} D \mu_{b}+\operatorname{Tr}\left\{D \Sigma_{b c}\right\}, \\
\text { s.t. } \nu-\delta(D) \leqslant 0, \\
\nu^{*} \nu+k^{*} k^{*}\left\{\delta(D)^{*} \delta(D)-\gamma(D)^{*} \gamma(D)\right\} \leqslant 0, \quad \nu \geqslant 0 .
\end{gathered}
$$

Finally, a solution, $D^{*}$, to this problem can be used to make nonparametric interval estimates for the value of the optimal objective function using (3) or simplifications (5) or (6), much as in the numerical example in the preceding section.

This variance can be used as the objective function in another model proposed by [3]

$$
\operatorname{Min} V\left[c^{\prime} x\right] \text { s.t. } \operatorname{Pr}[A x \leqslant b] \geqslant \alpha, \quad x=D b,
$$

and its deterministic equivalent which follows the development of (14).

A "partitioned" stochastic programming model considers the problem

$$
\operatorname{Max} c^{\prime} x+d^{\prime} y, \quad \text { s.t. } A x+B y \leqslant b ;
$$

where $y$ is a set of external, uncontrollable prior random variables, $d$ a vector of random prices, and $B$ a random technological coefficient matrix. By using the moments of $y, d, B$, and $b$, the problem may be restated

$$
\operatorname{Max} c^{\prime} x+c_{0}, \quad \text { s.t. } A x \leqslant b_{0} .
$$

with the moments of $b_{0}$ and $c_{0}$ specified exactly, and solved as previously shown.

\section{Conclusion}

Estimation of stochastic vector, or matrix, products may be virtually impossible by use of multivariate density functions and statistical transformations without severe and unrealistic simplifying assumptions about the random variates involved. Simulation can lead to approximations for a given problem, but at high computation cost and specificity for results.

However, an approach using multivariate moments, which are available from either empirical data or analytic densities, leads to exact statements about the mean and variance of the vector products and useful Tchebycheff-type probability estimates. These estimates are very helpful in generalizing the mathematical models of operations research.

\section{Appendix}

Derivation of Expressions for the Mean and Variance of the Inner Product of Two Vectors

$$
q=b^{\prime} c ; \quad q=\left(\tilde{b}+\mu_{b}\right)^{\prime}\left(\tilde{c}+\mu_{c}\right) ; \quad q=\tilde{b}^{\prime} \tilde{c}+2 \tilde{b}^{\prime} \mu_{c}+2 \mu_{b}^{\prime} \tilde{c}+\mu_{b}^{\prime} \mu_{c}
$$

Noting that $E(\tilde{b})=E(\tilde{c})=0$;

$$
\begin{aligned}
& E(q)=E\left(\tilde{b}^{\prime} \tilde{c}\right)+\mu_{b}^{\prime} \mu_{c} ; \quad E(q)=\mu_{b}^{\prime} \mu_{c}+\operatorname{Tr} \Sigma_{b c} \\
& V(q)=E\left(q^{2}\right)-[E(q)]^{2} ; \quad V(q)=E\left(b^{\prime} c\right)^{2}-\left(\mu_{b}^{\prime} \mu_{c}\right)^{2}-2 \mu_{b}^{\prime} \mu_{c} \operatorname{Tr} \Sigma_{b c}-\left(\operatorname{Tr} \Sigma_{b c}\right)^{2} .
\end{aligned}
$$


In terms of moments about the mean,

$$
\begin{aligned}
E\left(b^{\prime} c\right)^{2}= & E\left[\left(\tilde{b}+\mu_{b}\right)^{\prime}\left(\tilde{c}+\mu_{c}\right)\right]^{2} ; \\
E\left(b^{\prime} c\right)^{2}= & E\left[\tilde{b^{\prime}} \tilde{c} \tilde{c}^{\prime} \tilde{b}+\mu_{b}^{\prime} \tilde{c} \tilde{c}^{\prime} \mu_{b}+\mu_{c}^{\prime} \tilde{b} \tilde{b}^{\prime} \mu_{c}+\left(\mu_{b}^{\prime} \mu_{c}\right)^{2}+2 \mu_{b}^{\prime} \tilde{c} \tilde{c}^{\prime} \tilde{b}\right. \\
& +2 \mu_{c}^{\prime} \tilde{b} \tilde{b}^{\prime} \tilde{c}+2 \mu_{b}^{\prime} \mu_{c} \tilde{b}^{\prime} \tilde{c}+2 \mu_{b}^{\prime} \tilde{c} \tilde{b}^{\prime} \mu_{c}+2 \mu_{c}^{\prime} \tilde{b} \mu_{b}^{\prime} \mu_{c} \\
& \left.+2 \mu_{b}^{\prime} \tilde{c} \mu_{b}^{\prime} \mu_{c}\right] .
\end{aligned}
$$

We note that

$$
E\left(\tilde{c} \tilde{c}^{\prime}\right)=\Sigma_{c c} ; \quad E\left(\tilde{b} \tilde{b}^{\prime}\right)=\Sigma_{b b} ; \quad E\left(\tilde{b}^{\prime} \tilde{c}\right)=\operatorname{Tr} \Sigma_{b c} ; \quad E(\tilde{b})=E(\tilde{c})=0 .
$$

Hence,

$$
\begin{aligned}
E\left(b^{\prime} c\right)^{2}= & E\left(\tilde{b}^{\prime} \tilde{c} \tilde{c}^{\prime} \tilde{b}\right)+\mu_{b}^{\prime} \Sigma_{c c} \mu_{b}+\mu_{c}^{\prime} \Sigma_{b b} \mu_{c}+2 \mu_{b}^{\prime} \Sigma_{c b} \mu_{c}+\left(\mu_{b}^{\prime} \mu_{c}\right)^{2}+2 \mu_{b}^{\prime} E\left(\tilde{c} \tilde{c}^{\prime} \tilde{b}\right) \\
& +2 \mu_{c}^{\prime} E\left(\tilde{b} \tilde{b}^{\prime} \tilde{c}\right)+2 \mu_{b}^{\prime} \mu_{c} \operatorname{Tr} \Sigma_{b c} .
\end{aligned}
$$

We have

$$
\begin{aligned}
\mu^{\prime} \Sigma^{*} \mu & =\mu_{b}^{\prime} \Sigma_{c c} \mu_{b}+\mu_{c}^{\prime} \Sigma_{b b} \mu_{c}+2 \mu_{b}^{\prime} \Sigma_{c b} \mu_{c} \\
V(q) & =E\left(\tilde{b^{\prime}} \tilde{c} \tilde{c}^{\prime} \tilde{b}\right)+\mu^{\prime} \Sigma^{*} \mu+2 \mu_{c}^{\prime} E\left(\tilde{b} \tilde{b}^{\prime} \tilde{c}\right)+2 \mu_{b}^{\prime} E\left(\tilde{c} \tilde{c}^{\prime} \tilde{b}\right)-\left(\operatorname{Tr} \Sigma_{b c}\right)^{2} .
\end{aligned}
$$

\section{References}

1. Anderson, T., An Introduction to Multivariate Statistical Analysis, John Wiley and Sons, New York, 1958.

2. CAMP, B. H., "A New Generalization of Tchebycheff's Statistical Inequality," Bulletin of the American Mathematical Society, Vol. 28 (1922), p. 427.

3. Charnes, A. AND CoOper, W., "Deterministic Equivalents for Optimizing and Satisficing under Chance Constraints," Operations Research, Vol. 11, No. 1, (1963), pp. 18-39.

4. Godwin H. J., "On Generalization of Tchebycheff's Inequality," Journal of the American Statistical Society, Vol. 50 (1955), p. 923.

5. Graves, G. And Whinston, A., "An Algorithm for the Quadratic Assignment Problem," Management Science, Vol. 17, No. 7 (1970) pp. 453-471.

6. Howard, R., Dynamic Probabilistic Systems, Vol. 1, John Wiley and Sons, New York, 1971.

7. Jagannathan, R., "Chance Constrained Programming with Joint Constraints," Operations Research, Vol. 22, No. 2 (1974), pp. 358-372.

8. King, E., Sampson, C. And Simms, L., "An Alternative to Monte Carlo Sampling in Statistical Models," Management Science, Vol. 21, No. 6 (1975), p.649.

9. Mallows, C. C., "Generalization's of Tchebycheff's Inequalities," Journal of the Royal Statistical Society, Series B, Vol. XVIII, No. 2 (1956), p. 139.

10. Miller, B. And Wagner, H., "Chance-Constrained Programming with Joint Constraints," Operations Research, Vol. 13, No. 5 (1965), pp. 930-945.

11. Olkin, I. ANd PRatr. J., "A Multivariate Tchebycheff Inequality," Annals of Mathematical Statistics, Vol. 29 (1958), pp. 226-234.

12. Searle, S. R., Linear Models, John Wiley and Sons, New York, 1971.

13. Sengupta, J., Stochastic Programming Methods and Applications, North-Holland Publishing Company, New York, 1973.

14. —_- "Chance-Constrained Linear Programming with Chi-Square Type Deviates," Maragement Science, Vol. 19, No. 3 (1972), pp. 337-349.

15. Tintner, G., "A Note on Stochastic Linear Programming," Econometrica, Vol. 38, No. 2 (1960), pp. $490-495$. 